\title{
A Quantum Battle of the Sexes in Noninertial Frame
}

\author{
Guofu Weng, Yang Yu \\ National Laboratory of Solid State Microstructures, School of Physics, Nanjing University, \\ Nanjing, China \\ Email: physicswgf@126.com
}

Received 4 April 2014; revised 1 May 2014; accepted 26 May 2014

Copyright (C) 2014 by authors and Scientific Research Publishing Inc.

This work is licensed under the Creative Commons Attribution International License (CC BY).

http://creativecommons.org/licenses/by/4.0/

(c) (i) Open Access

\begin{abstract}
We investigate the influence of the Unruh effect on the quantum Battle of the Sexes (BOF). We show that the acceleration of a noninertial frame greatly affects the payoffs of the players. In the case of Battle of the Sexes, the Nash Equilibra in the inertial frame may be equilibrium but in other games this conclusion may not be true anymore. Furthermore, we also show that the new Nash Equilibria of the game played in the noninertial frame and the probability distributions of the players in the new Nash Equilibria vary with the acceleration of moving player and the degree of entanglement in the initial state shared by the players.
\end{abstract}

\section{Keywords}

\section{Quantum Game, Battle of the Sexes, Noninertial Frame}

\section{Introduction}

The classical game theory emerged from the work of Von Neumann [1], and is applied to various fields, such as economics, social science, medical science, biology and physics [2]-[4]. The relationship of the mathematical game theory and the sources of quantum entanglement was first reported in 1999 by David Meyer [5]. In the same year, Eisert and his coworkers proposed the quantization of nonzero sum games, and exploited the quantized scheme into studying Prisoners’ Dilemma as a particular case of game [6]. From then on the interested physicists have strenuously studied the field that is taken to be a completely new direction in quantum correlations and quantum computation processing. The original protocol for $2 \times 2$ quantum games was introduced by Eisert et al. through quantum entanglement [6] [7]. In the seminal paper of Meyer [5] and a number of subsequent papers [8]-[13] a particular two-parameter subset of $\mathrm{SU}(2)$ is introduced as the strategic space for the players, which has mathematical simplicity and can solve some of the dilemmas of classical game theory. 
The quantum entanglement between spatially separated parties is the powerful source of processing the quantum information and the quantum computation. At present, the behavior of quantum entanglement in relativistic setup is under exploration [14]-[17] and some fascinating conclusions have been received. The undergoing studies in the noninertial frames show that entanglement between different modes of various field is degraded by the Unruh effect and the degree of entanglement asymptotically attains a non-vanishing minimum value in the infinite acceleration. Recently, the relativistic setup has been applied to the study of quantum game in noninertial frame and some interesting results have been gained in references [18]-[20]. On the quantum Prisoner's Dilemma, for instance, S. Khan and M. K. Khan show that the payoff functions of the players are strongly affected by the acceleration of the noninertial frame and the symmetry of the game is disturbed in some extent [18]. Furthermore, quantum Stackelberg Duopoly in a Noninertial frame has been investigated and found that benefit of the initial state entanglement in the quantum form of the duopoly in the initial frame is adversely influenced by the acceleration of the non-stationary player [19]. Some theorists generalized the quantum game for three players, which one of the players stays stationary and the two others move with a uniform acceleration [21].

Quantum game theorists have studied various specific games, such as Prisoner's Dilemma, Battle of the Sexes, Cournot duopoly, Stackelberg Duopoly, and symmetric games like Rock-Paper-Scissors and so on [8]-[13] and [18]-[20] and [22]-[24]. Researchers intended to find solutions to those classical game in the background of quantum entanglement. Here, the word solution refers to a set of strategies that the rational players will surely play [22].

In this Letter, we study the influence of the Unruh effect on the payoff functions of the players in the Battle of Sexes. We find that the game in noninertial frame can be transformed to classical game when the initial state is unentangled and both players remain stationary (i.e. acceleration is zero). We show that the expected payoffs of both players are relevant not only to the probability distributions of the players and the degree of entanglement of the initial state but they also vary with the acceleration of the moving player.

\section{Quantum Version of Battle of the Sexes in Noninertial Frame}

We exploit the concepts of quantum games in noninertial frame to study a two-person static game of complete information, which is called the Battle of the Sexes. The game is usually described as the following exposition: A women, Alice, and a man, Bob, are planning to decide where to spend the Saturday night: Alice would prefer to attend the Ballet, while Bob would like to watch the football match at the television. They would prefer to stay together rather than far apart. We can represent the game in a normal form by denoting by $\mathbf{B}$ (Ballet) and $\mathbf{F}$ (Football) the two pure strategies which constitutes the common strategic space $\mathcal{S}$. The game is represented by the payoff matrix in the Table 1.

\subsection{Quantization Scheme}

In order to quantize the classical strategies $\mathbf{B}$ and $\mathbf{F}$ for player Alice, we assigned two basic vectors $|0\rangle$ and $|1\rangle$ in a Hilbert space of two-level system to $\mathbf{B}$ and $\mathbf{F}$, respectively [6]. Similarly quantization scheme can be applied to the strategies of player Bob and we obtain the quantum formation of the payoff table in the Table 2.

\subsection{The Quantum Game in Noninertial Frame}

We consider that Alice and Bob share an entangled initial state $\left|\psi_{i}\right\rangle=\hat{J}|00\rangle$ of the two qubits at a point in flat Minkowski spacetime. Alice and Bob possess the first entry and the second entry in the ket, respectively. the unitary operator $\hat{J}$ is symmetric for fair games. The players perform their strategic moves as local unitary operators $\hat{U}(\theta, \alpha, \beta)$ belonging to a full space of SU(2) strategies, which is a set of three-parameter strategies:

$$
\hat{U}(\theta, \alpha, \beta)=\left(\begin{array}{cc}
\mathrm{e}^{i \alpha} \cos \left(\frac{\theta}{2}\right) & \mathrm{e}^{i \beta} \sin \left(\frac{\theta}{2}\right) \\
\mathrm{e}^{-i \beta} \sin \left(\frac{\theta}{2}\right) & \mathrm{e}^{-i \alpha} \cos \left(\frac{\theta}{2}\right)
\end{array}\right),
$$


Table 1. Payoff matrix for the classical Battle of Sexes. The first entry in a pair of numbers denotes the payoff of Alice and the second entry stand for Bob’s payoff.

\begin{tabular}{ccc}
\hline & Bob:B & Bob:F \\
\hline Alice:B & $(\alpha, \beta)$ & $(\gamma, \gamma)$ \\
Alice:F & $(\gamma, \gamma)$ & $(\beta, \alpha)$ \\
\hline
\end{tabular}

Table 2. The quantization scheme by the Eisert protocol: classical pure strategies B (Ballet) and $\mathrm{F}$ (Football) correspond to the quantum pure strategies $|0\rangle$ and $|1\rangle$, respectively.

\begin{tabular}{ccc} 
& $|0\rangle$ & $|1\rangle$ \\
\hline$|0\rangle$ & $|00\rangle$ & $|01\rangle$ \\
$|1\rangle$ & $|10\rangle$ & $|11\rangle$ \\
\hline
\end{tabular}

where $\theta \in[0, \pi], \alpha, \beta \in\left[0, \frac{\pi}{2}\right]$.

With classical strategic moves, $\hat{D}=\hat{U}(\pi, 0,0)=\left(\begin{array}{cc}0 & 1 \\ -1 & 0\end{array}\right), \hat{I}=\hat{U}(0,0,0)=\left(\begin{array}{ll}1 & 0 \\ 0 & 1\end{array}\right)$.

The unitary operator $\hat{J}$ is an entangling operator and then is given by

$$
\hat{J}=\exp \left[i \frac{\delta}{2} \hat{D} \otimes \hat{D}\right],
$$

where $\delta \in[0, \pi / 2]$ and is a measure of the degree of entanglement in the initial state. The initial state is maximally entangled for the upper limit of $\delta$ and has no entanglement for the lower limit of $\delta$.

The entangling operators $\hat{J}$ must be known to both players for the knowledge of the degree of the entanglement in the initial state. After the application of the entangling operator, the state of the game evolves to

$$
\left|\psi_{i}\right\rangle=\cos \frac{\delta}{2}|00\rangle+i \sin \frac{\delta}{2}|11\rangle .
$$

We assume that Alice stays stationary and Bob moves with a uniform acceleration. Each player possesses a device which is sensitive only to a single mode in their respective regions. From the accelerated player Bob's frame, the Minkowski vacuum state is given by a two-mode squeezed state,

$$
|0\rangle_{M}=\cos r|0\rangle_{I}|0\rangle_{I I}+\sin r|1\rangle_{I}|1\rangle_{I I},
$$

where $\cos r=\left(\mathrm{e}^{-2 \pi \omega c / a}+1\right)^{-1 / 2}$. The parameter $r$ is the dimensionless parameter, which $r=0$ when $a=0$ and $r=\pi / 4$ when $a=\infty$. The constants $\omega, c$, and $a$, in the above equation stand, respectively, for the Dirac particle's frequency, light speed in vacuum and Bob's acceleration. In the Equation (4),the subscripts $I$ and II of the kets represent the Rindler modes in the region I and II, respectively, in the Rindler spacetime diagram Figure 1. The Equation (4) represents that the noninertial observer that moves with a constant acceleration in region $I$ observes a thermal state instead of the vacuum state, which is called Unruh effect [25] [26].

The excited state in Minkowski spacetime is relevant to Rindler modes as follows:

$$
|1\rangle_{M}=|1\rangle_{I}|0\rangle_{I I}
$$

In terms of Minkowski mode for Alice and Rindler modes for Bob, the entangled initial state of Equation (3) by exploiting Equations (4) and (5) evolves

$$
|\psi\rangle_{A, I, I I}=\cos \frac{\delta}{2} \cos r|0\rangle_{A}|0\rangle_{I}|0\rangle_{I I}+\cos \frac{\delta}{2} \sin r|0\rangle_{A}|1\rangle_{I}|1\rangle_{I I}+i \sin \frac{\delta}{2}|0\rangle_{A}|1\rangle_{I}|0\rangle_{I I}
$$

The corresponding mixed density matrix in the entangled initial state in Equation (6) is defined as

$$
\rho_{A, I, I I}=|\psi\rangle_{A, I, I I}\left\langle\left.\psi\right|_{A, I, I I}\right.
$$




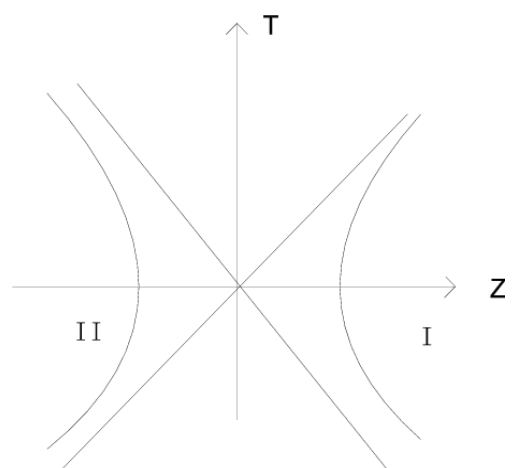

Figure 1. Rindler spacetime diagram: The uniformly accelerated observer Bob (B) moves on a hyperbola with constant acceleration a in the region $I$ and is causally disconnected from the region $I I$ which a fictitious observer anti-Bob $(\hat{B})$ moves on.

A uniformly accelerated observer (Bob) in Rindler region $I$ is causally disconnected from the other region at the opposite side Figure 1, therefore, we must take trace over all the mode in region II. After the trace eperation, the density matrix is expressed as

$$
\begin{aligned}
\rho_{A, I}= & \cos ^{2} \frac{\delta}{2} \cos ^{2} r|00\rangle\left\langle 00\left|+\cos ^{2} \frac{\delta}{2} \sin ^{2} r\right| 01\right\rangle\langle 01| \\
& +i \sin \frac{\delta}{2} \cos \frac{\delta}{2} \cos r|11\rangle\left\langle 00\left|-i \sin \frac{\delta}{2} \cos \frac{\delta}{2} \cos r\right| 00\right\rangle\langle 11|, \\
& +\sin ^{2} \frac{\delta}{2}|11\rangle\langle 11|
\end{aligned}
$$

Note that the subscripts of the kets and bras have been dropped for simplicity. The density matrix can also be written as the following matrix:

$$
\rho_{A, I}=\left(\begin{array}{cccc}
\cos ^{2} \frac{\delta}{2} \cos ^{2} r & 0 & 0 & -i \sin \frac{\delta}{2} \cos \frac{\delta}{2} \cos r \\
0 & \cos ^{2} \frac{\delta}{2} \sin ^{2} r & 0 & 0 \\
0 & 0 & 0 & 0 \\
i \sin \frac{\delta}{2} \cos \frac{\delta}{2} \cos r & 0 & 0 & \sin ^{2} \frac{\delta}{2}
\end{array}\right)
$$

In the quantum Battle of the Sexes, each player has two possible strategies $I$, the identity operator, and $\sigma$, the inversion operator or the Pauli's bit-flip operator. Let $p$ and $1-p$ represent the probabilities of $I$ and $\sigma$ that Alice applies. Similarly, $q$ and $1-q$ are the probabilities that Bob applies the identity operator and inversion operator, respectively. After the players take their strategic moves, the final density matrix evolves to

$$
\begin{aligned}
\rho_{f}= & p q I_{A} \otimes I_{B} \rho_{A, 1} I_{A}^{\dagger} \otimes I_{B}^{\dagger}+p(1-q) I_{A} \otimes \sigma_{B} \rho_{A, 1} I_{A}^{\dagger} \otimes \sigma_{B}^{\dagger} \\
& +q(1-p) \sigma_{A} \otimes I_{B} \rho_{A, 1} \sigma_{A}^{\dagger} \otimes I_{B}^{\dagger}+(1-p)(1-q) \sigma_{A} \otimes \sigma_{B} \rho_{A, I} \sigma_{A}^{\dagger} \otimes \sigma_{B}^{\dagger}
\end{aligned}
$$

The payoff operators for Alice and Bob can be written as

$$
\begin{aligned}
& P_{A}=\alpha|00\rangle\langle 00|+\gamma(|01\rangle\langle 01|+| 10\rangle\langle 10|)+\beta| 11\rangle\langle 11| \\
& P_{B}=\beta|00\rangle\langle 00|+\gamma(|01\rangle\langle 01|+| 10\rangle\langle 10|)+\alpha| 11\rangle\langle 11|
\end{aligned}
$$

The payoff functions for Alice and Bob in the Battle of the Sexes respectively, are

$$
\overline{\$}_{A}=\operatorname{Tr}\left[P_{A} \rho_{f}\right] ; \quad \overline{\$}_{B}=\operatorname{Tr}\left[P_{B} \rho_{f}\right]
$$


For the sake of convenience, the following variables can be defined:

$$
\begin{aligned}
& x_{1} \equiv \alpha \cos ^{2} \frac{\delta}{2} \cos ^{2} r+\gamma \cos ^{2} \frac{\delta}{2} \sin ^{2} r+\beta \sin ^{2} \frac{\delta}{2} \\
& x_{2} \equiv \gamma \cos ^{2} \frac{\delta}{2} \cos ^{2} r+\alpha \cos ^{2} \frac{\delta}{2} \sin ^{2} r+\gamma \sin ^{2} \frac{\delta}{2} \\
& x_{3} \equiv \gamma \cos ^{2} \frac{\delta}{2} \cos ^{2} r+\beta \cos ^{2} \frac{\delta}{2} \sin ^{2} r+\gamma \sin ^{2} \frac{\delta}{2} \\
& x_{4} \equiv \beta \cos ^{2} \frac{\delta}{2} \cos ^{2} r+\gamma \cos ^{2} \frac{\delta}{2} \sin ^{2} r+\alpha \sin ^{2} \frac{\delta}{2}
\end{aligned}
$$

By using the above variables, the payoff functions in Equation (13) can be expressed in the following succinct way:

$$
\begin{aligned}
& \overline{\$}_{A}(p, q, \delta, \gamma)=p q x_{1}+p(1-q) x_{2}+q(1-p) x_{3}+(1-p)(1-q) x_{4} \\
& \overline{\$}_{B}(p, q, \delta, \gamma)=p q x_{4}+p(1-q) x_{3}+q(1-p) x_{2}+(1-p)(1-q) x_{1}
\end{aligned}
$$

The payoff functions in Equation (18) show the expected gains of both players depend on not only the probability distributions $\{p, 1-p\}$ and $\{q, 1-q\}$ for Alice and Bob respectively, but also on the degree of entanglement $\delta$ and dimensionless acceleration parameter $r$. Furthermore, even in the case of unentangled initial state (i.e. $\delta=0$ ), the expected payoff functions depend on the probability distributions of both players and as well vary with the the acceleration $a$ of Bob.

Before we continue, let us first find some relationships about the variables defined in Equations (14)-(17):

$$
x_{4}-x_{2}=(\alpha+\beta-2 \gamma) \cos ^{2} \frac{\delta}{2} \cos ^{2} r+2(\gamma-\alpha) \cos ^{2} \frac{\delta}{2}+(\alpha-\gamma)
$$

The value of $x_{4}-x_{2}$ is not always positive. It could be nonnegative for some domains of $\delta$ and $r$ since $\gamma-\alpha \leq 2(\gamma-\alpha) \cos ^{2} \frac{\delta}{2} \leq 0$ is true for the fact that $0 \leq \delta \leq \frac{\pi}{2}$. For instance $x_{4}-x_{2} \geq 0$, when $\delta=\pi / 2$ and $0 \leq r \leq \frac{\pi}{4}$

For the same sake, we can obtain the following inequalities:

$$
\begin{gathered}
x_{4}-x_{3}=(\beta-\gamma) \cos ^{2} \frac{\delta}{2} \cos 2 r+(\alpha-\gamma) \sin ^{2} \frac{\delta}{2} \geq 0 \\
x_{1}-x_{2}=(\alpha-\gamma) \cos ^{2} \frac{\delta}{2} \sin 2 r+(\beta-\gamma) \sin ^{2} \frac{\delta}{2} \geq 0 \\
x_{1}-x_{3}=(\alpha+\beta-2 \gamma) \cos ^{2} \frac{\delta}{2} \cos ^{2} r+(\beta-\gamma)\left(\sin ^{2} \frac{\delta}{2}-\cos ^{2} \frac{\delta}{2}\right) \geq 0
\end{gathered}
$$

For the definite degree of entanglement $\delta$ and the acceleration of Bob, the expected payoff functions can be rewritten as follows:

$$
\overline{\$}_{A}(p, q, \delta, \gamma) \equiv \overline{\$}_{A}(p, q), \overline{\$}_{B}(p, q, \delta, \gamma) \equiv \overline{\$}_{B}(p, q) .
$$

Nash Equilibria can be found by imposing the two coupled inequalities:

$$
\begin{array}{ll}
\overline{\$}_{A}\left(p^{*}, q^{*}\right)-\overline{\$}_{A}\left(p, q^{*}\right)=\left(p^{*}-p\right)\left[q^{*}\left(x_{1}+x_{4}-x_{2}-x_{3}\right)+x_{2}-x_{4}\right] \geq 0 & \forall p \in[0,1], \\
\overline{\$}_{B}\left(p^{*}, q^{*}\right)-\overline{\$}_{B}\left(p^{*}, q\right)=\left(q^{*}-q\right)\left[p^{*}\left(x_{1}+x_{4}-x_{2}-x_{3}\right)+x_{2}-x_{1}\right] \geq 0 & \forall q \in[0,1] .
\end{array}
$$

\section{Results and Analysis}

When two factors in both inequalities are both the same sign, we can get the Nash Equilibrium. There are three 
possibilities:

Case 1. $p_{1}^{*}=1, q_{1}^{*}=1$. In such a case both Alice and Bob play the pure strategy $\mathbf{B}$ (Ballet). their expected payoff functions are $\overline{\$}_{A}(1,1)=x_{1}$ and $\overline{\$}_{B}(1,1)=x_{4}$, and by employing the in equalities (20) and (22) the following inequalities hold:

$$
\begin{aligned}
& \overline{\$}_{A}(1,1)-\overline{\$}_{A}(p, 1)=(1-p)\left(x_{1}-x_{3}\right) \geq 0 \quad \forall p \in[0,1], \\
& \overline{\$}_{B}(1,1)-\overline{\$}_{B}(1, q)=(1-q)\left(x_{4}-x_{3}\right) \geq 0 \quad \forall q \in[0,1] .
\end{aligned}
$$

The payoff functions in Nash Equilibriua are relevant to the degree of entanglement of the initial state and the dimensionless acceleration parameter $r$. In inertial frame, the parameter $r=0$, the payoffs of both players coincide when the initial state is in maximally entangled. In this particular situation, the gains of both players are $\overline{\$}_{A}(1,1)=\overline{\$}_{B}(1,1)=(\alpha+\beta) / 2$ respectively. In the noninertial frame, the payoff function, taking the gain of Alice for instance, is strongly influenced by the acceleration parameter $r$ such that the gain of Alice is given by $\overline{\$}_{A}(1,1)=\alpha \cos ^{2} \frac{\delta}{2} \cos ^{2} r+\gamma \cos ^{2} \frac{\delta}{2} \sin ^{2} r+\beta \sin ^{2} \frac{\delta}{2}$, which becomes $\overline{\$}_{A}(1,1)=\frac{1}{2}\left(\alpha \cos ^{2} \frac{\delta}{2}+\gamma \cos ^{2} \frac{\delta}{2}\right)+\beta \sin ^{2} \frac{\delta}{2}$ when player Bob is assumed to move in an infinite acceleration corresponding to the parameter $r=\pi / 4$.

Case 2. $p_{2}^{*}=0, q_{2}^{*}=0$. In this case both Alice and Bob play the another pure strategy $\mathbf{F}$ (Football). Their expected payoff functions are reversed with respect to the previous case: $\overline{\$}_{A}(0,0)=x_{4}$ and $\overline{\$}_{B}(0,0)=x_{1}$. Meanwhile, by the equation (19) we get following truth:

The payoff function of Alice

$$
\overline{\$}_{A}(0,0)-\overline{\$}_{A}(p, 0)=p\left(x_{4}-x_{2}\right) \geq 0 \quad \forall p \in[0,1]
$$

is not always nonnegative for the nonpositive-definite value of $x_{4}-x_{2}$. In such a case this pure strategy is not the Nash Equilibrium when the value of $x_{4}-x_{2}$ is negative. The new Nash equilibria could arise when $x_{4}-x_{2}$ is positive by choosing the degree of entanglement in the initial state and the acceleration of Bob.

By inequality (21) we get the following inequality for the payoff function of Bob:

$$
\overline{\$}_{B}(0,0)-\overline{\$}_{B}(0, q)=q\left(x_{1}-x_{2}\right) \geq 0 \quad \forall q \in[0,1] .
$$

In the inertial frame $(r=0)$, the payoff functions are $\overline{\$}_{A}(0,0)=\alpha \cos ^{2} \frac{\delta}{2}+\beta \sin ^{2} \frac{\delta}{2}$ and

$\overline{\$}_{B}(0,0)=\beta \cos ^{2} \frac{\delta}{2}+\alpha \sin ^{2} \frac{\delta}{2}$, respectively. If two players share an unentangled initial state $(\delta=0)$, the gains of the players in noninertial frame are $\overline{\$}_{A}(0,0)=\alpha \cos ^{2} r+\gamma \sin ^{2} r$ and $\overline{\$}_{B}(0,0)=\beta \cos ^{2} r+\gamma \sin ^{2} r$, which are relevant to the dimensionless acceleration parameter $r$.

Case 3. When the probabilities $p$ and $q$ are less than one but greater than zero, two of the factors in inequalities (24) and (25) should be reasonable to be zero. Hence, we can solve the probabilities:

$$
p_{3}^{*}=\frac{x_{1}-x_{2}}{x_{1}+x_{4}-x_{2}-x_{3}} \text { and } q_{3}^{*}=\frac{x_{4}-x_{2}}{x_{1}+x_{4}-x_{2}-x_{3}}
$$

which correspond to the gains of both Alice and Bob coincide:

$$
\overline{\$}_{A}\left(p^{*}, q^{*}\right)=\overline{\$}_{B}\left(p^{*}, q^{*}\right)=\frac{x_{1} x_{4}-x_{2} x_{3}}{x_{1}+x_{4}-x_{2}-x_{3}}
$$

The new Nash Equilibria are relevant to acceleration of Bob and degree of entanglement in the initial state for the value of $x_{4}-x_{2}$. When the value of $x_{4}-x_{2}$ is negative, the probability distribution of Bob is meaningless and it is so far from the purpose that we could get new Nash Equilibrium.

In the case of unentangled initial state (i.e. $\delta=0$ ), the Equations (14)-(17) are reduced into the following equations: 


$$
\begin{array}{ll}
x_{1}=\alpha \cos ^{2} r+\gamma \sin ^{2} r & x_{2}=\gamma \cos ^{2} r+\alpha \sin ^{2} r \\
x_{3}=\gamma \cos ^{2} r+\beta \sin ^{2} r & x_{4}=\beta \cos ^{2} r+\gamma \sin ^{2} r
\end{array}
$$

By using these equations, we can receive that $x_{1}+x_{4}-x_{2}-x_{3}=(\alpha+\beta-2 \gamma) \cos 2 r$ and $x_{1} x_{4}-x_{2} x_{3}=\left(\alpha \beta-\gamma^{2}\right) \cos 2 r$, by which the gains of players are obtained:

$\overline{\$}_{A}\left(p^{*}, q^{*}\right)=\overline{\$}_{B}\left(p^{*}, q^{*}\right)=\frac{\alpha \beta-\gamma^{2}}{\alpha+\beta-2 \gamma}$.

\section{Discussions and Conclusions}

As we can find from Eqautions (14)-(17) that $x_{1}=\alpha, x_{2}=\gamma, x_{3}=\gamma$, and $x_{4}=\beta$ when the initial state is unentangled and both Alice and Bob are in the inertial frame (i.e. $a=0$ ). In such case, the payoff functions for players correspond to the classical game and their expected payoffs are:

$$
\begin{aligned}
& \overline{\$}_{A}(p, q)=p[q(\alpha+\beta-2 \gamma)+\gamma-\beta]+\beta+q(\gamma-\beta), \\
& \overline{\$}_{B}(p, q)=q[p(\alpha+\beta-2 \gamma)+\gamma-\beta]+\alpha+p(\gamma-\alpha)
\end{aligned}
$$

In this classical mixed strategies, three Nash Equilibria can be found [22]: 1) probability distribution ( $p_{1}^{*}=1$, $q_{1}^{*}=1$ ) corresponds to pure strategy that both Alice and Bob choose to play $\mathbf{B}$. Their expected payoff functions are $\overline{\$}_{A}(1,1)=\alpha$ and $\overline{\$}_{B}(1,1)=\beta$. 2) Strategies $p_{2}^{*}=0$ and $q_{2}^{*}=0$ correspond to pure strategy, both Alice and Bob play $\mathbf{F}$, and expected payoffs are $\overline{\$}_{A}(0,0)=\beta$ and $\left.\overline{\$}_{B}(0,0)=\alpha .3\right) \quad p_{3}^{*}=\frac{\alpha-\gamma}{\alpha+\beta-2 \gamma}$ and $q_{3}^{*}=\frac{\beta-\gamma}{\alpha+\beta-2 \gamma}$, which are correctly larger than zero and less than one, correspond to a Nash Equilibrium for which the payoffs of both players coincide: $\overline{\$}_{A}\left(p_{3}^{*}, q_{3}^{*}\right)=\overline{\$}_{B}\left(p_{3}^{*}, q_{3}^{*}\right)=\frac{\alpha \beta-\gamma^{2}}{\alpha+\beta-2 \gamma}$.

In a summary, we have shown that payoff functions are strongly influenced by the acceleration of moving player Bob in the noninertial frame. Payoff functions are affected by Unruh effect in the quantum Battle of the Sexes in noninertial frame. In the case of Battle of Sexes, the Nash Equilibria in inertial frame are still equilibria in the corresponding noninertial frame but the payoffs of both Alice and Bob are relevant to both degree of entanglement in the players' initial state and the acceleration parameter. In the case of probability distribution $\left\{p_{3}^{*}, q_{3}^{*}\right\}$, it is a new Nash Equilibrium for the probability distributions of both play are related to the degree of entanglement and acceleration parameter, which can be backward induced to the inertial situation when we suppose the dimensionless accelerating parameter $r=0$. In the limit of infinite acceleration, we find that the payoff functions at the Nash Equilibria would be irrelevant to the acceleration but only depend on the entanglement.

\section{Acknowledgements}

This work is supported by the National Key Projects for Basic Researches of China (No. 2011CB922104, 2011CBA00200), by the National Natural Science Foundation of China (No. 91021003, 11274156), the Natural Science Foundation of Jiangsu Province(No. BK2010012), and PAPD.

\section{References}

[1] von Neumann, J. (1951) Applied Mathematical Series, 3, 36-38.

[2] von Neumann, J. and Morgenstern, O. (1944) Theory of Games and Economic Behavior. Princeton University Press, Princeton.

[3] Piotrowski, E.W. and Sładkowski, J. (2002) Physica A: Statistical Mechanics and Its Applications, 312, $208-216$.

[4] Baaquie, B.E. (2001) Physics Review E, 64, Article ID: 016121. http://dx.doi.org/10.1103/PhysRevE.64.016121 
[5] Meyer, D.A. (1999) Physics Review Letter, 82, 1052-1055. http://dx.doi.org/10.1103/PhysRevLett.82.1052

[6] Eiset, J., Wilkens, M. and Lewenstein, M. (1999) Physics Review Letter, 83, 3077-3080. http://dx.doi.org/10.1103/PhysRevLett.83.3077

[7] Eisert, J. and Wilkins, M. (2000) Journal of Modern Optics, 47, 2543-2556. http://dx.doi.org/10.1080/09500340008232180

[8] Iqbal, A. and Toor, A.H. (2001) Physics Letter A, 280, 249-256. http://dx.doi.org/10.1016/S0375-9601(01)00082-2

[9] Du, J., Li, H., Xu, X., Shi, M., Wu, J. and Han, R. (2002) Physics Review Letter, 88, Article ID: 1379021. http://dx.doi.org/10.1103/PhysRevLett.88.137902

[10] Chen, L.K., Ang, H., Kiang, D., Kwek, L.C. and Lo, C.F. (2003) Physics Letter A, 316, 317-323. http://dx.doi.org/10.1016/S0375-9601(03)01175-7

[11] Özdemir, S.K., Shimamura, J. and Imoto, N. (2004) Physics Letter A, 325, 104-111. http://dx.doi.org/10.1016/j.physleta.2004.03.042

[12] Özdemir, S.K., Shimamura, J., Morikoshi, F. and Imoto, N. (2004) Physics Letter A, 333, 218-231. http://dx.doi.org/10.1016/j.physleta.2004.10.055

[13] Alonso-Sanz, R. (2012) Proceedings of the Royal Society A, 468, 3370-3383. http://dx.doi.org/10.1098/rspa.2012.0161

[14] Alsing, P.M., Fuentes-Schuller, I., Mann, R.B. and Tessier, T.E. (2006) Physics Review A, 74, Article ID: 032326. http://dx.doi.org/10.1103/PhysRevA.74.032326

[15] Terashima, H. and Ueda, M. (2003) International Journal of Quantum Information, 1, 93. http://dx.doi.org/10.1142/S0219749903000061

[16] Pan, Q. and Jing, J. (2008) Physics Review A, 77, Article ID: 024302. http://dx.doi.org/10.1103/PhysRevA.77.024302

[17] Fuentes-Schuller, I. and Mann, R.B. (2005) Physics Review Letter, 95, Article ID: 120404. http://dx.doi.org/10.1103/PhysRevLett.95.120404

[18] Khan, S. and Khan, M.K. (2011) Journal of Physics A: Mathematical and Theoretical, 44, Article ID: 355302. http://dx.doi.org/10.1088/1751-8113/44/35/355302

[19] Khan, S. and Khan, M.K. (2011) Chinese Physics Letters, 28, Article ID: 070202. http://dx.doi.org/10.1088/0256-307X/28/7/070202

[20] Khan, S. and Khan, M.K. (2013) Quantum Information Process, 12, 1351-1353. http://dx.doi.org/10.1007/s11128-012-0469-5

[21] Goudarzi, H. and Beyrami, S. (2012) Journal of Physics A: Mathematical and Theoretical, 45, Article ID: 225301. http://dx.doi.org/10.1088/1751-8113/45/22/225301

[22] Marinatto, L. and Weber, T. (2000) Physics Letter A, 272, 291-303. http://dx.doi.org/10.1016/S0375-9601(00)00441-2

[23] Iqbal, A. and Toor, A.H. (2004) Communications in Theoretical Physics, 42, 335-338.

[24] Iqbal, A. and Toor, A.H. (2002) Physics Review A, 65, Article ID: 022306. http://dx.doi.org/10.1103/PhysRevA.65.022306

[25] Davies, P.C.W. (1975) Journal of Physics A: Mathematical and General, 8, 609-616. http://dx.doi.org/10.1088/0305-4470/8/4/022

[26] Unruh, W.G. (1976) Physics Review D, 14, 870-892. http://dx.doi.org/10.1103/PhysRevD.14.870 
Scientific Research Publishing (SCIRP) is one of the largest Open Access journal publishers. It is currently publishing more than 200 open access, online, peer-reviewed journals covering a wide range of academic disciplines. SCIRP serves the worldwide academic communities and contributes to the progress and application of science with its publication.

Other selected journals from SCIRP are listed as below. Submit your manuscript to us via either submit@scirp.org or Online Submission Portal.
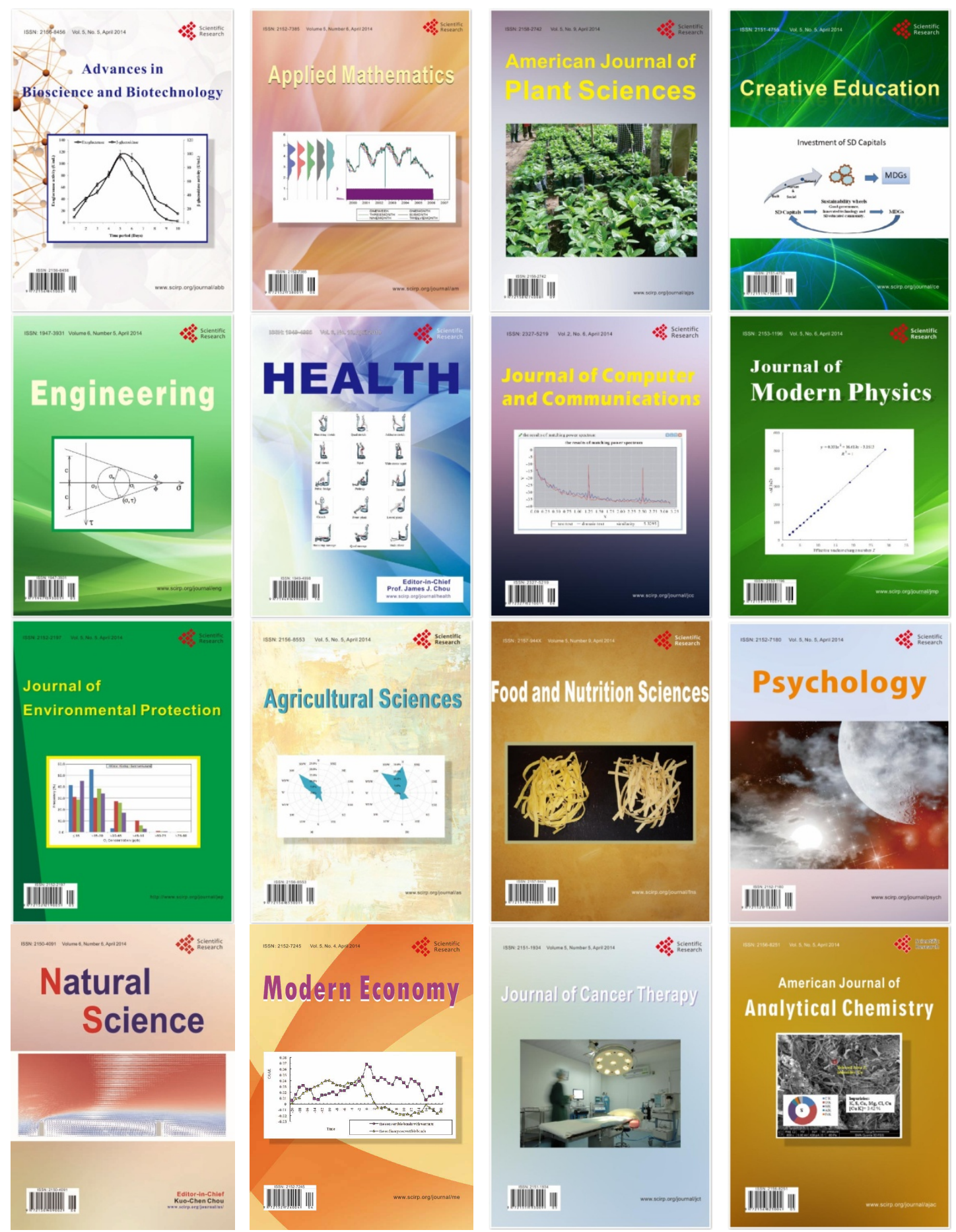